\title{
Simulation of Selected Traveling Wave Directional Multiplexers
}

\author{
Engr. M. S. ALGHAMDI
}

B.S. in Electrical and Computer Engineering, Electrical and Computer Engineering Department, King Abdul-Aziz University, Jeddah, Saudi Arabia

\section{Prof. A. M. AFFANDI}

Professor of Electrical and Computer Engineering, Electrical and Computer Engineering Department, King Abdul-Aziz University, Jeddah, Saudi Arabia

\begin{abstract}
:
Simulation of selected traveling wave multiplexers have been done in this paper in order to obtain the desired results based on the existing theoretical analysis on this field. The even and odd mode analysis have been added in the beginning to show case the expected desired conclusions made by the simulation software. The equivalent circuits of the even and odd modes are given. An optimization program has been utilized in order to optimize the multiplexer's findings. Four multiplexers with different bandwidths have been simulated and tested by via ADS program to highlight the returns and validity of the modified traveling wave multiplexers.
\end{abstract}

Keywords: Traveling Wave, Directional Multiplexers, Simulation 


\section{Introduction}

A communication channel for transmission in any communication system is required, whether it's digital or analog. A wired or wireless connection can be used for this channel. Individual channels for each user would be impractical. As a result, a collection of signals is merged and broadcast over a single channel. We employ multiplexers to achieve this. Multiplexing is the process of combining data from multiple sources into a single signal and transmitting it through a common medium. Multiplexing has the advantage of allowing us to send more signals across a single media. This channel, which might be a physical medium such as a coaxial, metallic conductor or a wireless link, must handle several signals at the same time (S. Coale,1956).

As a result, transmission costs can be decreased. Even if the transmissions are on the same channel, they do not always happen at the same time. These devices are well-suited for use as frequency division multiplexers. The most essential property that distinguishes directional filters from other channel separators such as duplexes and circulators is that they work very well at all ports and across a wide frequency range, which makes them very useful. Franklin S. Coale presented a microwave multiplexer based on a traveling wave directional filter (S. Coale,1956), (Standley, 1963).

The ability of this network to offer a response in the form of a travelling wave rather than the traditional form of a standing wave is its distinguishing feature. This circuit has a constant resistance circuit and a well-matched input port as a result of the suggested network (provide a very low VSWR). While Robert D. Standley looked at the impact of loop discontinuity and loop side characteristic impedance on the performance of traveling wave directional filters (Standley, 1963).

The approximate and exact synthesis techniques that can be used to compute the travelling wave directional filter parameters were disclosed by John L.B. (Walker,1978). A good approach of studying the TWDM has been proposed by G. Reiter. (Reiter,1990). The TWDM was employed as a time delay triggered trapatt oscillator by B.H. Newton, P.L. Booth, and R. Davies (Newton and others,1983) A proposed Traveling wave directional 
filters designs are simulated and the results of each are obtained by utilizing the Advanced System Software (ADS).

\section{Circuit Analysis}

The four-port network of the modified travelling wave directional filter (MTWDF) is shown in Figure 1. The symmetry lines are also depicted in the same figure. The scattering matrix of the proposed network is as follows:

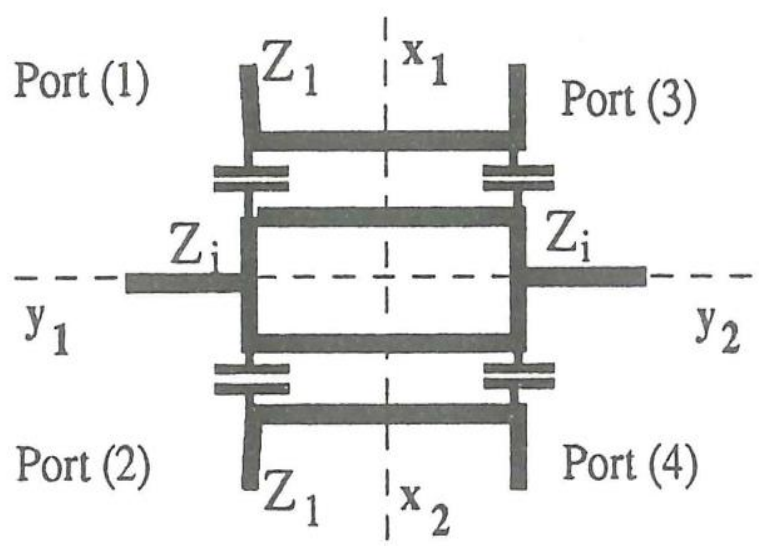

Figure 1: The MTWDF multiplexer

$\left[\begin{array}{c}\mathrm{V}_{1^{-}} \\ \mathrm{V}_{2^{-}} \\ \mathrm{V}_{3^{-}} \\ \mathrm{V}_{4^{-}}\end{array}\right]=\left[\begin{array}{llll}\mathrm{S}_{11} & \mathrm{~S}_{12} & \mathrm{~S}_{13} & \mathrm{~S}_{14} \\ \mathrm{~S}_{21} & \mathrm{~S}_{22} & \mathrm{~S}_{23} & \mathrm{~S}_{24} \\ \mathrm{~S}_{31} & \mathrm{~S}_{32} & \mathrm{~S}_{33} & \mathrm{~S}_{34} \\ \mathrm{~S}_{41} & \mathrm{~S}_{42} & \mathrm{~S}_{43} & \mathrm{~S}_{44}\end{array}\right]\left[\begin{array}{c}\mathrm{V}_{1^{+}} \\ \mathrm{V}_{2^{+}} \\ \mathrm{V}_{3^{+}} \\ \mathrm{V}_{4^{+}}\end{array}\right]$

Figure 2: Scattering Matrix of 4-port network

Where V1-, V2-, V3- and V4- are the reflected voltages at ports (1), (2), (3) and (4) respectively, while $\mathrm{V} 1+, \mathrm{V} 2+, \mathrm{V} 3+$ and $\mathrm{V} 4+$ are the incident voltages at ports (1), (2), (3) and (4) respectively. Also, the scattering matrix at the central frequency can be expressed as:

$$
\left[\begin{array}{c}
\mathrm{V}_{1^{-}} \\
\mathrm{V}_{2^{-}} \\
\mathrm{V}_{3^{-}} \\
\mathrm{V}_{4^{-}}
\end{array}\right]=\left[\begin{array}{llll}
0 & 1 & 0 & 0 \\
1 & 0 & 0 & 0 \\
0 & 0 & 0 & 1 \\
0 & 0 & 1 & 0
\end{array}\right]\left[\begin{array}{c}
\mathrm{V}_{1^{+}} \\
\mathrm{V}_{2^{+}} \\
\mathrm{V}_{3^{+}} \\
\mathrm{V}_{4^{+}}
\end{array}\right]
$$

Figure 3: Scattering matrix at the central frequency 
Academic Journal of Research and Scientific Publishing | Vol 3 | Issue 29

Publication Date: 5-9-2021

When the input signal is used at port (1), the scattering matrix at the center frequency shows that the output port (2) operates as a band pass filter, while the output at port (3) or (4) serves as a bandreject filter.

\section{THEORETICAL ANALYSIS OF THE MODIFIED TRAVELLING WAVE}

\section{DIRECTIONAL FILTERS (M. T. W. D. F.)}

Using the even-odd mode principles, there are several approaches for assessing the M.T.W.D.F. (S. Coale, 1956), (Reiter, 1990). The modified travelling wave directional filter with its line of symmetries X1X2 and Y1Y2 is shown in Fig. 4, while the even-odd mode equivalent circuit are shown in Fig. 5a, 5b.

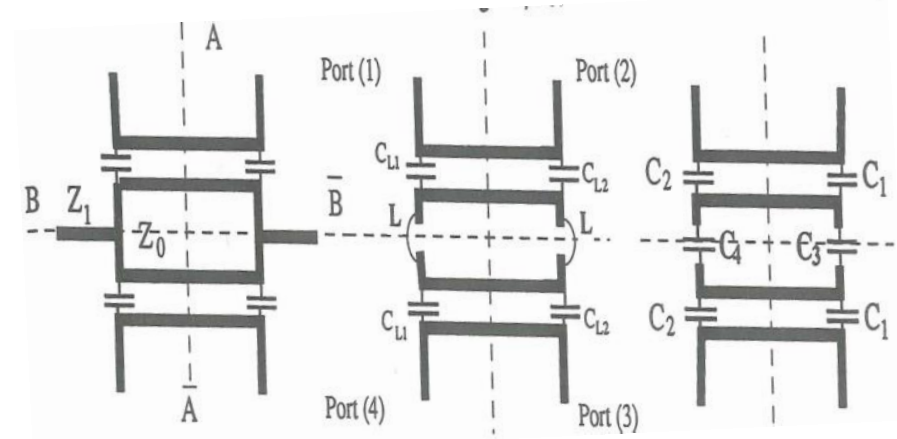

Figure 4: The MTWDF with its Line of Symmetry

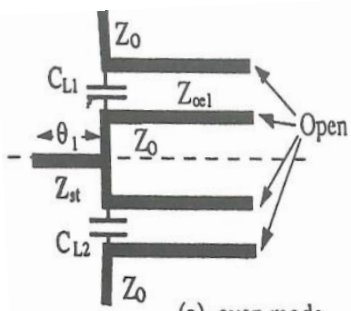

(a) even mode

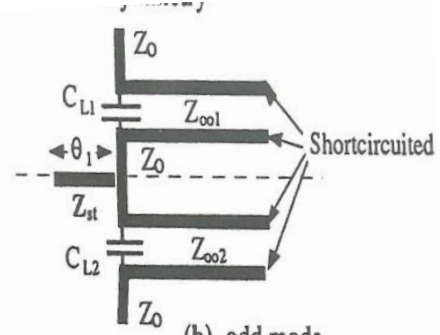

(b) odd mode

Figure 5: The even-odd mode equivalent circuit of MTWDM with stubs 
The derivation of The Modified Travelling Wave Directional Filter (MTWDF) is done using The even and odd mode equivalent circuits depicted in Figures $6 \mathrm{a}$ and $6 \mathrm{~b}$ which can be used to calculate the even and odd mode chain matrices. The even and odd mode parameters can be simply determined once these matrices have been found. The overall parameters of the proposed network can then be derived using the superposition theorem. Figures $5 \mathrm{a}, 5 \mathrm{~b}$ are equivalent to Figures $6 \mathrm{a}$, 6b demonstrated below:

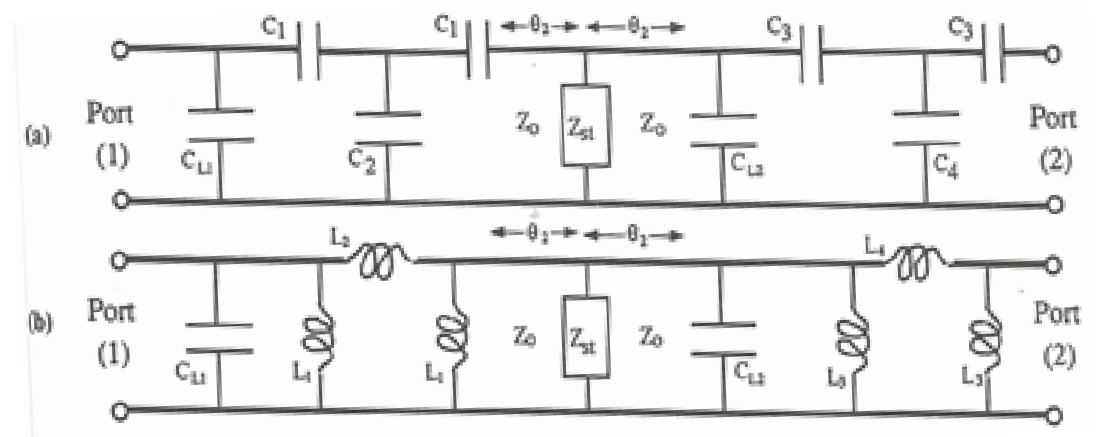

Figure 6: the even and odd mode of MTWDM

Using Fig. 6a, 6b, the even and odd mode chain matrix can be found as:

$$
\left[\begin{array}{cc}
A_{e, o} & B_{e, o} \\
C_{e, o} & D_{e, o}
\end{array}\right]=\left[M_{1} e, o\right] *\left[M_{2}\right] *\left[M_{3} e, o\right]
$$

Where the subscript e,o refers to even and odd mode respectively, while $Z_{s}=-j Z_{1} \tan \theta_{1}$ for the distributed stub, $\mathrm{j} \omega \mathrm{L}_{\mathrm{s}}$ for wire inductance and $-\mathrm{j} / \omega \mathrm{C}_{\mathrm{s}}$ for the capacitance respectively. The values for the matrices of (M) can be expressed as:

$$
\mathrm{M} 1 e=\left[\begin{array}{cc}
1 & \frac{1}{S C_{1}} \\
S C L_{1} & 2
\end{array}\right]\left[\begin{array}{cc}
1 & \frac{1}{S C_{1}} \\
S C_{2} & \frac{S C_{2}}{S C_{1}}+1
\end{array}\right]=\left[\begin{array}{cc}
1+\frac{S C_{2}}{S C_{1}} & \frac{1}{S C_{1}}+\frac{1}{S C_{1}}\left(\frac{S C_{2}}{S C_{1}}+1\right) \\
S C L_{1}+2 S C_{2} & \frac{S C L_{1}}{S C_{1}}+2\left(\frac{S C_{2}}{S C_{1}}+1\right)
\end{array}\right]
$$




$$
\mathrm{M} 2=\left[\begin{array}{cc}
\operatorname{Cos} \theta_{2}+j Y_{S 10} Z_{0} \operatorname{Sin} \theta_{2} & j Z_{0} \operatorname{Sin} \theta_{2} \\
j Y_{0} \operatorname{Sin} \theta_{2}+Y_{s 10} \operatorname{Cos} \theta_{2} & \operatorname{Cos} \theta_{2}
\end{array}\right]=\left[\begin{array}{cc}
A & B \\
C & D
\end{array}\right]
$$

Where,

$$
\begin{aligned}
& \mathrm{A}=\operatorname{Cos} \theta_{2}\left[\operatorname{Cos} \theta_{2}+j Y_{s 10} Z_{0} \operatorname{Sin} \theta_{2}\right]-Z_{0} Y_{0} \operatorname{Sin}^{2} \theta_{2} . \\
& \mathrm{B}=j Z_{0} \operatorname{Sin} \theta_{2}\left[\operatorname{Cos} \theta_{2}+j Y_{s 10} Z_{0} \operatorname{Sin} \theta_{2}\right]+j Z_{0} \operatorname{Sin} \theta_{2} \operatorname{Cos} \theta_{2} . \\
& \mathrm{C}=\operatorname{Cos} \theta_{2}\left[j Y_{0} \operatorname{Sin} \theta_{2}+Y_{s 10} \operatorname{Cos} \theta_{2}\right]+j Y_{0} \operatorname{Sin} \theta_{2} \operatorname{Cos} \theta_{2} . \\
& \mathrm{D}=j Z_{0} \operatorname{Sin} \theta_{2}\left[j Y_{0} \operatorname{Sin} \theta_{2}+Y_{s 10} \operatorname{Cos} \theta_{2}\right]+\operatorname{Cos}^{2} \theta_{2} .
\end{aligned}
$$

$\mathrm{M} 3 e=$

$$
\left[\begin{array}{crr}
1+\frac{S C_{4}}{S C_{3}} & \frac{1}{S C_{3}}+\frac{1}{S C_{3}}\left(\frac{S C_{4}}{S C_{3}}+1\right) \\
S C L_{1}+S C_{4} & \left(\frac{S L_{2}}{S C_{3}}+1\right) & \frac{S L_{2}}{S C_{3}}+\left(\frac{S L_{2}}{S C_{3}}+1\right)\left(\frac{S C_{2}}{S C_{1}}+1\right)
\end{array}\right]
$$

$$
M_{1 o}=
$$

$$
\left[\begin{array}{cr}
1+\frac{S L_{2}}{S L_{1}} & S L_{2} \\
\left(S L_{1}+\frac{1}{S L_{1}}\right)\left(1+\frac{S L_{2}}{S L_{1}}\right)+\frac{1}{S L_{1}} & S L_{2}\left(S C L_{1}+\frac{1}{S L_{1}}\right)+1
\end{array}\right]
$$

The values of the capacitances and inductances can be taken from reference (Jshii and Ozaki, 1958), (Mitra and Temes, 1973). and then modified to be used in microstrip.

$$
\text { M } 3{ }_{0}=\left[\begin{array}{lr}
1+\frac{S L_{4}}{S L_{3}} & S L_{4} \\
\left(S L_{2}+\frac{1}{S L_{3}}\right)\left(1+\frac{S L_{4}}{S L_{3}}\right)+\frac{1}{S L_{3}} & S L_{4}\left(S C L_{2}+\frac{1}{S L_{3}}\right)+1
\end{array}\right]
$$

Now the even and odd mode reflection and transmission coefficients are expressed respectively 


$$
\begin{gathered}
\rho_{e, o}=\frac{A_{e, o}+Z_{L}+B_{e, o}-Z_{0} Z_{L} C_{e, o}-Z_{0} D_{e, o}}{A_{e, o}+Z_{L}+B_{e, o}-Z_{0} Z_{L} C_{e, o}+Z_{0} D_{e, o}} \\
\tau_{e, o}=\frac{A e, o+Z_{L}+B_{e, o}-Z_{0} Z_{L} C_{e, o}-Z_{0} D_{e, o}}{A e, o+Z_{L}+B_{e, o}-Z_{0} Z_{L} C_{e, o}+Z_{0} D_{e, o}}
\end{gathered}
$$

Applying the superposition theorem, on the derived even and odd parameters, therefore the overall parameters of the modified TWDM can be determined. If the input signal is introduced at port (1) of the TWDM, the outputs at TWDM ports are:

Output at port $(\mathbf{1})=\mathrm{S} 11=1 / 2 \rho e+1 / 2 \rho o$

Output at port $(2)=\mathrm{S} 21=1 / 2 \rho e+1 / 2 \rho o$

Output at port $(\mathbf{3})=\mathrm{S} 31=1 / 2 \tau_{e}+1 / 2 \tau_{o}$

Output at port (4) $=\mathrm{S} 41=1 / 2 \tau_{e}+1 / 2 \tau_{o}$

The same procedures can be applied on the other parts of the TWDM to compute the rest of scattering matrix of the TWDM.

One of the goals of this study is to create a set of MTWDM multiplexers with different bandwidths, such as narrow, moderate, and large band multiplexers, with band-pass properties that are nearly mirror images of band-stop properties. After grasping the theory behind the functionality of traveling wave directional filters, our next step is the simulation of multiple designs in order to achieve the properties of the proposed filters, which is band-pass, and band-stop filters.

The expected behavior of desired results has been similar to the filters mentioned in this study which were band-pass and band-stop filters. As shown below:

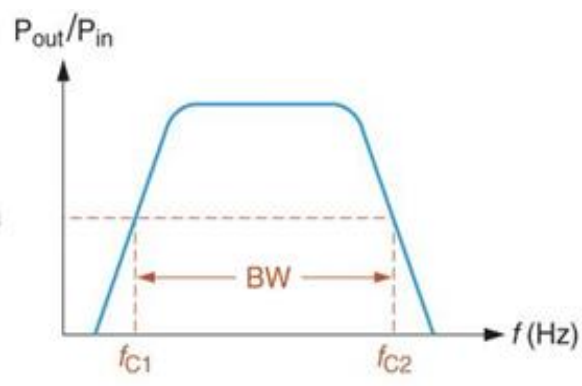

(a)

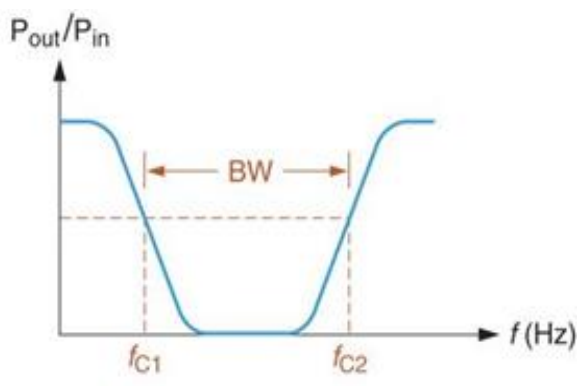

(b)

Figure 7: plot of (a) band-pass filter and (b) band-stop filter 
Academic Journal of Research and Scientific Publishing | Vol 3 | Issue 29

\section{DESIGNS SIMULATION AND RESULTS}
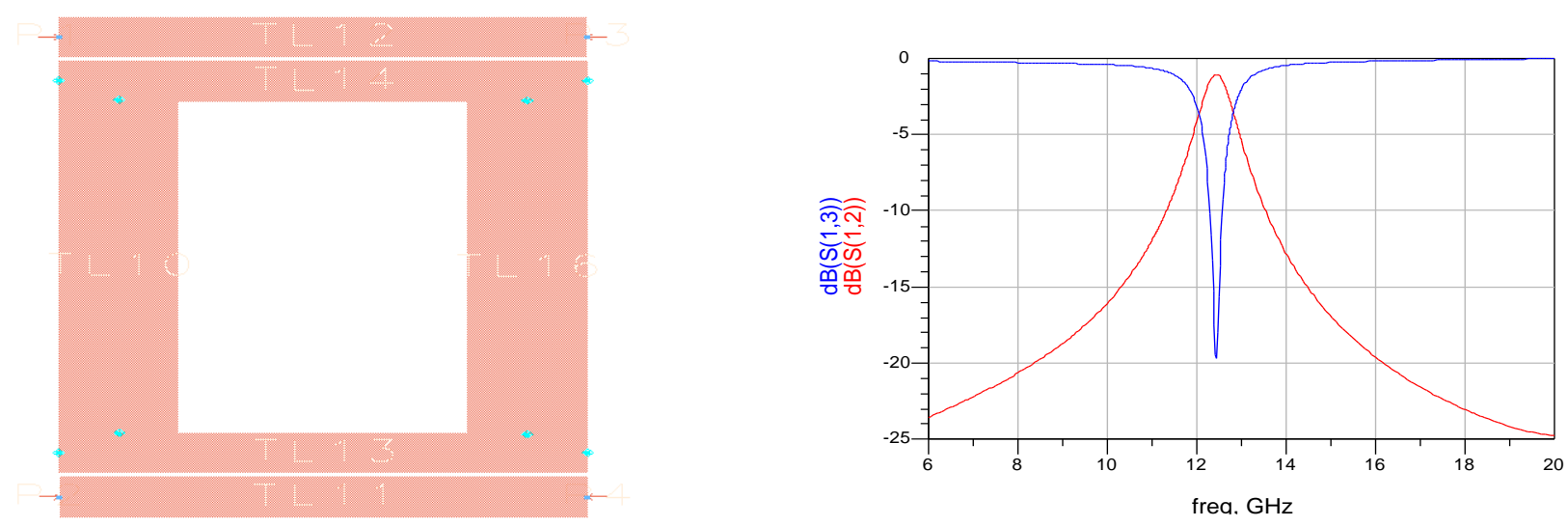

Figure 8: 4-port microstrip multiplexer

Figure 9: S-Parameter response of fig.8

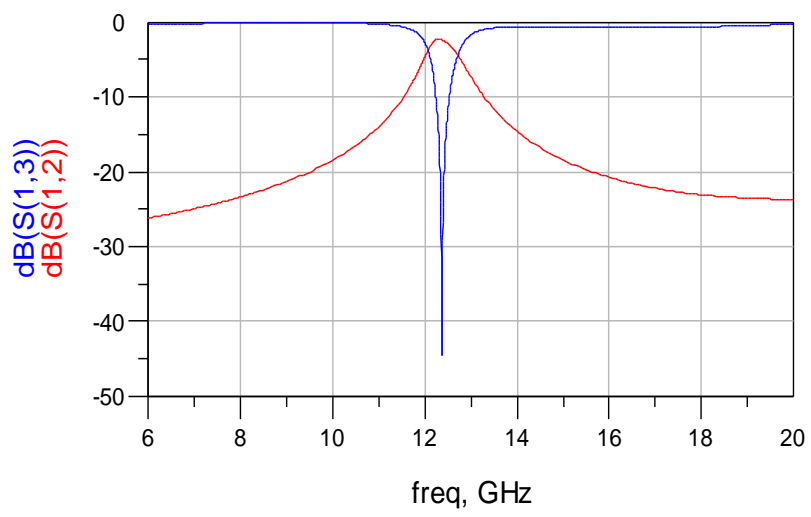

Figure 10: 4-port microstrip multiplexer

Figure 11: S-Parameter response of fig.10 with different geometry 


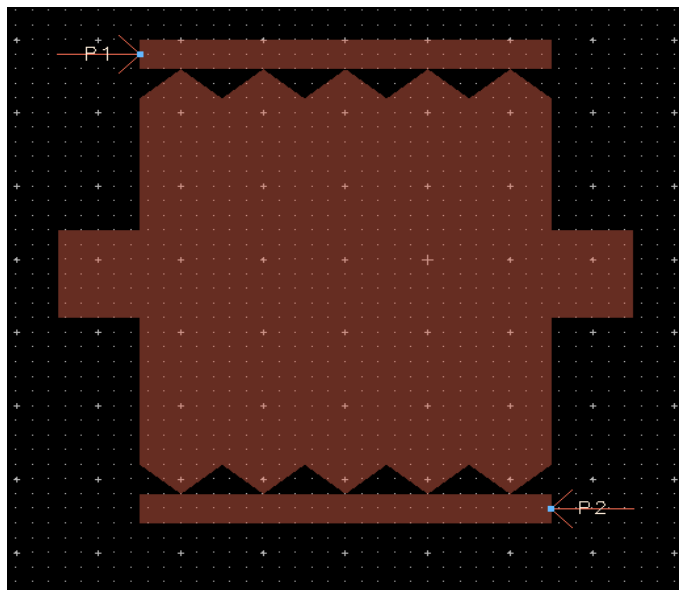

Figure 12: 2-port microstrip multiplexer with triangular spacing

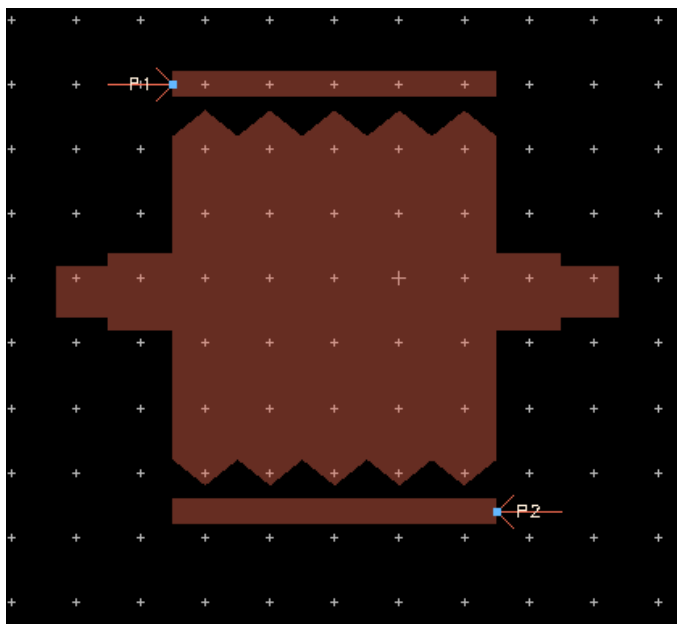

Figure 14: 2-port microstrip multiplexer with triangular spacing and added stub.

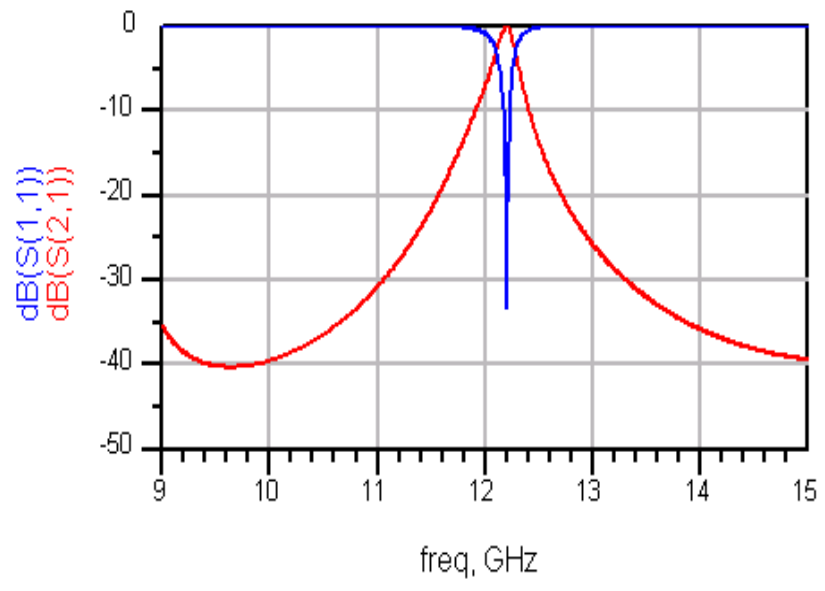

Figure 13: S-Parameter response of fig.12

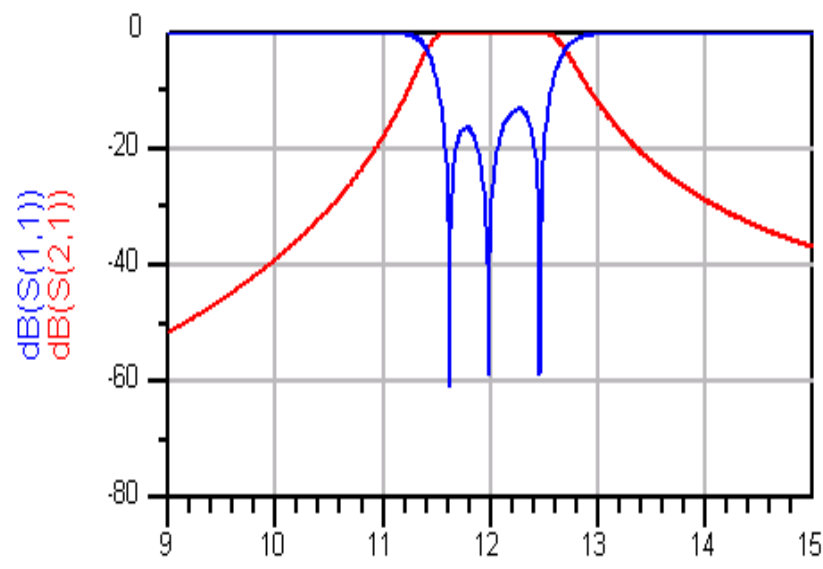

freq, $\mathrm{GHz}$ 
In the above designs the concept of modified travelling wave directional filters are implemented by utilizing a simulation software for microwave frequencies. The response of each design is a good representation of band-pass and band-reject filters depending on the criteria and desire of the application. These designs could be fabricated in the future to follow up the measured results with the simulation data. Microstrip circuits are easier (and less expensive) to fabricate than stripline, with less processing steps and easier placement of circuit components.

The effective dielectric material used throughout the simulation is Rogers RO3003 ${ }^{\mathrm{TM}}$ dielectric materials, where ceramic loading is used for stability, and are easier to fabricate for microstrip circuits than more costly RT/duroid 5880 materials. The thickness of the substrates ranges from $0.5 \mathrm{~mm}$ to $1.50 \mathrm{~mm}$ in these designs, the layouts shown in the figures above are done arbitrary by trial and error to reach the desired S-parameter results.

\section{Conclusion}

There are many times in life when it is necessary or desirable to send several signals at the same time, or when the application itself requires multiple signals. In any case, using communication channels to deliver numerous information signals can save money and time. The capacity to combine them is one of the most essential functions of the travelling wave directional filter. Different designs of traveling wave directional filters were developed and simulated using the advanced system software (ADS). Employing the theoretical concepts of the past studies of the even and odd mode and the expected desired results obtained and measured of the mentioned references is in agreement to the calculations obtained by the simulation tools. Four designs are shown in the simulation part, consisting of two-port and four-port networks functioning as traveling wave directional couplers. The microstrip characteristics, dimensions, and geometry are manipulated to enhance and improve the results.

\section{References:}

S. Coale, Franklin (1956), "A Travelling Wave Directional Filter", IRE Trans, on Microwave Theory and Tech., Vol. MTT-4, pp. 250-260.

Standley, Robert D (1963), Frequency Response of Strip Line Travelling Wave Directional Filter", IRE Trans, on Microwave Theory and Tech., Vol. 11, pp. 264-265. 
Standley, Robert D (1963), "Discontinuity Effect in Single Resonator Travelling Wave Filter", IRE Trans, on Microwave Theory and Tech., Vol. 11, pp. 551-552.

Walker, John L.B (1978), "Exact and Approximate Synthesis of TEM-Mode Transmission Type Directional Filters", IEEE Trans, on Microwave and Tech., Vol. MTT26, No. 3.

G. Reiter (1990), "A Method to Design Microstrip Directional Filters", European Conference, Vol. 2, pp. 1073-1078.

Newton and others (1983), "Time Delay Triggered Trapatt Oscillator with Directional Filter", IEEE Trans, on Microwave Theory and Tech., Vol. MTT-31, No. 5, pp. 423.

Jshii and Ozaki (1958), "Synthesis of a Class of Strip-Line Filters", IRE Trans, on Circuit Theory, pp. 104-109.

Mitra and Temes (1973), "Modern Filter Theory and Design", John Willey \& Sons, pp. 192-230.

Miley, J.E (1974), "Looking for a 3 to 8 dB Microstrip Coupler", Microwave, pp. 5863.

https://electricalacademia.com/electronics/band-pass-notch-filter-circuit-theory/

Affandi, A. M. (1995), "A Modified Version of Traveling Wave Directional Multiplexers", The Fourth Engineering Saudi Conference, VOLUME III.

Copyright @ 2021 Engr. M. S. ALGHAMDI, Prof. A. M. AFFANDI, AJRSP. This is an open-access article distributed under the terms of the Creative Commons Attribution License (CC BY NC). 